Article

\title{
An Early Decrease in Release of Aquaporin-2 in Urinary Extracellular Vesicles After Cisplatin Treatment in Rats
}

\author{
Hiroko Sonoda, Sayaka Oshikawa-Hori and Masahiro Ikeda *( \\ Department of Veterinary Pharmacology, Faculty of Agriculture, University of Miyazaki, Miyazaki 889-2192, \\ Japan; sonoda-h@cc.miyazaki-u.ac.jp (H.S.); oshikawa.sayaka.g3@cc.miyazaki-u.ac.jp (S.O.-H.) \\ * Correspondence: a0d302u@cc.miyazaki-u.ac.jp; Tel.: +81-985-58-7268
}

Received: 30 December 2018; Accepted: 8 February 2019; Published: 10 February 2019

check for updates

\begin{abstract}
Aquaporin-1 (AQP1) and AQP2 are important proteins involved in the regulation of renal water handling. Both AQPs have been found in urinary extracellular vesicles (uEVs) (uEV-AQP1 and -AQP2). Cisplatin, an antineoplastic agent, is known to down-regulate renal AQP1 and AQP2. However, the effect of cisplatin on the release of uEV-AQP1 and -AQP2 is largely unknown. In this study, we examined whether treatment of rats with cisplatin affected the release of uEV-AQP1 and -AQP2. Blood tests indicated that renal function was little altered at $24 \mathrm{~h}$ after cisplatin treatment but thereafter decreased dramatically at all of the other time points examined. Release of uEV-AQP1 was slightly increased at $24 \mathrm{~h}$ and decreased at $168 \mathrm{~h}$. On the other hand, release of uEV-AQP2 was decreased dramatically at $24 \mathrm{~h}$, and the decrease was maintained during the experimental period. These data suggest that uEV-AQP2 can be used to detect early renal impairment due to cisplatin. Furthermore, a combination of uEV-AQP2 and -AQP1 may be useful for estimation of cisplatin-induced renal injury in a stage-dependent manner.
\end{abstract}

Keywords: urinary extracellular vesicles; exosomes; aquaporin-1; aquaporin-2; cisplatin; acute kidney injury

\section{Introduction}

Aquaporins (AQPs) are membrane water channels, and at least 13 aquaporin isoforms (AQP0 to 12) have been identified in mammals. In the kidney, seven AQPs (AQP1, 2, 3, 4, 6, 7 and 11) are known to be expressed at distinct sites along the nephron [1]. Among them, AQP1 and AQP2 have been identified in the urine. Aquaporin-1 is expressed in the proximal tubule, descending thin limb, and descending vasa recta cells, contributing to water reabsorption in the proximal tubules and formation of a renal osmotic gradient. Aquaporin-2 is expressed mainly at the apical membrane and intracellular vesicles in the collecting duct principal cells. Apical membrane expression of AQP2 is known to be regulated by arginine vasopressin (AVP), an antidiuretic hormone [2].

Urinary extracellular vesicles (uEVs), tiny vesicles produced by renal epithelial cells [3,4], are mainly classified into two subsets including exosomes and microvesicles. Exosomes (30-100 nm in diameter) are released into urine by fusion of the outer membrane of multi-vesicular bodies with the apical plasma membrane of renal epithelial cells [5]. Microvesicles (100-1000 nm in diameter) are produced by a budding process of plasma membrane [5]. Aquaporin-1 and AQP2 in the urine have been reported to be localized to uEVs (uEV-AQP1 and uEV-AQP2), especially exosomes $[4,6,7]$.

We previously reported that $\mathrm{uEV-AQP1}$ and -AQP2 might reflect the expression levels of AQP1 and AQP2 in the proximal tubule and collecting duct cells, respectively $[8,9]$. Also, the release of uEV-AQP1 and -AQP2 was decreased in models of acute kidney injury (AKI) including gentamicinand renal ischemia-induced injury models in rats [8-10]. 
Cisplatin is a widely used chemotherapeutic agent administered to patients with testicular cancer, ovarian germ cell tumors, and head and neck cancer [11]. However, therapeutic use of cisplatin is associated with toxicity resulting in renal injury [12-14]. The proximal tubule in the kidney is known to be the principal site for renal damage due to cisplatin [12-14]. It has also been reported that cisplatin treatment causes necrosis of the distal tubules and collecting ducts [12-14]. Although the molecular mechanisms underlying cisplatin-induced nephrotoxicity have not been fully elucidated, the toxicity is thought to be mediated through its interaction with nuclear DNA, mitochondrial DNA and other mitochondrial targets. For example, it has been reported that mitochondria in the renal inner medullary collecting duct cells are injured by cisplatin $[15,16]$.

Previous studies using rats have indicated that renal expression of AQP1 and AQP2 is decreased by treatment with cisplatin, resulting in a defect in the urinary concentration mechanism $[17,18]$. However, it remains unclear whether cisplatin affects the release of uEV-AQP1 and -AQP2. In the present study, we examined the effect of cisplatin on the release of uEV-AQP1 and-AQP2.

\section{Materials and Methods}

\subsection{Animal Models}

All animal studies were performed with approval from the University of Miyazaki in accordance with Guidelines for the Care and Use of Laboratory Animals in the University of Miyazaki.

Male Sprague-Dawley (SD) rats were purchased from Kyudo (Saga, Japan). All animals were kept in metabolic cages and given free access to water during the study period. Sprague-Dawley rats $(316-510 \mathrm{~g})$ were injected with cisplatin $(7.5 \mathrm{mg} / \mathrm{kg})$ (Sigma, Tokyo, Japan) intraperitoneally (the cisplatin group). This dose was selected because a previous report had indicated that cisplatin at this dose significantly affected the renal expression of AQPs [18]. Rats receiving only a vehicle (saline) injection served as the control group. Blood and urine samples were collected at $24 \mathrm{~h}$ (control, $\mathrm{n}=18$; cisplatin, $\mathrm{n}=18$; from 6 experiments), $72 \mathrm{~h}$ (control, $\mathrm{n}=22$; cisplatin, $\mathrm{n}=21$; from 7 experiments), $120 \mathrm{~h}$ (control, $\mathrm{n}=15$; cisplatin, $\mathrm{n}=14$; from 5 experiments), and $168 \mathrm{~h}$ (control, $\mathrm{n}=12$; cisplatin, $\mathrm{n}=12$; from 4 experiments), and kidney samples were obtained at $24 \mathrm{~h}$ (control, $\mathrm{n}=6$; cisplatin, $\mathrm{n}=6$; from 2 experiments), $72 \mathrm{~h}$ (control, $\mathrm{n}=10$; cisplatin, $\mathrm{n}=9$; from 3 experiments), $120 \mathrm{~h}$ (control, $\mathrm{n}=6$; cisplatin, $\mathrm{n}=5$; from 2 experiments), and $168 \mathrm{~h}$ (control, $\mathrm{n}=6$; cisplatin, $\mathrm{n}=7$; from 2 experiments) after treatment with either vehicle or cisplatin.

Plasma creatinine and urea nitrogen concentrations were measured by an autoanalyzer (FUJIDRICHEM, Fuji Film Medical Co., Ltd. Tokyo, Japan).

\subsection{Urine Collection and Isolation of $u E V s$}

Urine was collected for 6 h (5 a.m. to 11 a.m.; 24 h, 18-24 h; 72 h, 66-72 h; 120 h, 114-120 h; $168 \mathrm{~h}, 162-168 \mathrm{~h}$ after treatment) with a collection tube containing a protease inhibitor mixture (60 $\mu \mathrm{l}$ of $130 \mathrm{mM}$ EDTA, $70 \mathrm{mM}$ p-amidinophenyl methanesulfonyl fluoride hydrochloride, complete protease inhibitor cocktail tablet). The uEV-rich fraction was obtained using a urine sample whose volume had been adjusted by a certain amount of creatinine (e.g., $1 \mathrm{mg}$ creatinine) employing a sequential differential centrifugation technique $\left(1000 \times g\right.$ for $15 \mathrm{~min}$ at $\mathrm{RT}, 17,000 \times g$ for $15 \mathrm{~min}$ at $25^{\circ} \mathrm{C}, 200,000 \times g$ for $1 \mathrm{~h}$ at $25^{\circ} \mathrm{C}$ ) as described previously [8-10]. The pellet obtained after the final centrifugation was suspended in $50 \mu \mathrm{L}$ of a solution of the above protease inhibitor mixture diluted with MilliQ water. After the suspension was mixed with $4 \times$ sample buffer ( $8 \%$ SDS, 50\% glycerol, $250 \mathrm{mM}$ Tris- $\mathrm{HCl}$, $0.05 \%$ bromo phenol blue, $200 \mathrm{mM} \mathrm{DTT}$ ), the mixture was incubated for $30 \mathrm{~min}$ at $37^{\circ} \mathrm{C}$.

\subsection{Kidney Protein Extraction}

The kidney was divided into three regions, the cortex, outer medulla and inner medulla, under a stereoscopic microscope. Each region of kidney was homogenized in an ice-cold isolation solution (300 mM sucrose, 1.3 mM EDTA, $25 \mathrm{mM}$ imidazole, complete protease inhibitor cocktail tablet) for 
10 min using a shaker-type homogenizer at $1500 \mathrm{rpm}$ for $10 \mathrm{~min}$ (Shakemaster Neo, Bio Medical Science, Tokyo, Japan). The homogenate was centrifuged at $1000 \times g$ for $10 \mathrm{~min}$ at $4^{\circ} \mathrm{C}$, and the supernatant was subsequently ultra-centrifuged at $200,000 \times g$ for $1 \mathrm{~h}$. The pellet obtained from ultra-centrifugation was suspended in the isolation solution, and this suspension was mixed with $4 \times$ sample buffer. This mixture was thereafter incubated at $37^{\circ} \mathrm{C}$ for $30 \mathrm{~min}$. The protein concentration in a small amount of suspension solution from each pellet before addition of the sample buffer was determined using the Pierce BCA Protein Assay reagent Kit (Thermo Fisher Scientific Inc., Rockford, IL, USA).

\subsection{Immunoblot Analysis}

Immunoblot analysis was performed as previously described [8-10], using the following antibodies: Anti-AQP1 (cat no. sc-20810; Santa Cruz Biotechnology Inc., Santa Cruz, CA, USA), anti-AQP2 (cat no. AQP-002; Alomone Labs, Jerusalem, Israel), anti-GAPDH antibody (cat. no. sc-25778; Santa Cruz Biotechnology Inc.), and HRP-conjugated anti-rabbit IgG (cat no. 7074; Cell Signaling Technology, Danvers, MA, USA). Antibody-associated protein on the membrane was detected by Super Signal ${ }^{\circledR}$ chemiluminescence detection system (Thermo Fisher Scientific Inc.). The protein bands were visualized by a polaroid camera (GE Healthcare UK Ltd., Amersham, England) or a LAS4000 system (GE Healthcare UK Ltd.). The pictures taken by the camera were scanned using a scanner (GT-S650, Seiko Epson corp., Nagano, Japan) and the density of the band was quantified by the WinRoof software V5.7 (MITANI CORPORATION, Tokyo, Japan). The representative picture taken by the camera was shown after a monochrome inversion under the Adobe Photoshop CC 2017 software (ver 18.0.1, Adobe Systems Co., Ltd, Tokyo, Japan), while retaining the original quality. The resulting band visualized by the LAS4000 system was evaluated by a ImageQuant TL software (GE Healthcare UK Ltd.).

For preliminary validation of the GAPDH internal control, the levels of renal expression of GAPDH were compared between the control and cisplatin groups. The mean \pm standard error of the mean (SEM) values are shown in a supplementary table (Table S1), and the differences in values between the groups for the same region at each time point were not significantly different, indicating that GAPDH was appropriate as an internal control.

In each series of experiments, a control group comprising several animals was included. When immunoblotting analysis was performed, protein samples from the corresponding control animals were always loaded in each gel for normalization.

\subsection{Histology}

The paraffin-embedded kidney blocks (fixation with 10\% paraformaldehyde) were cut at $2 \mu \mathrm{m}$ thickness and the sections were stained with periodic acid-Schiff (PAS) reagent (Muto Pure Chemicals Co., Ltd., Tokyo, Japan).

For immunofluorescence staining, after retrieval of antigen by incubating specimen in distilled water at $121{ }^{\circ} \mathrm{C}$ for $5 \mathrm{~min}$, the specimens were immersed in a $3 \% \mathrm{H}_{2} \mathrm{O}_{2}$ solution to consume the endogenous peroxidase and then were blocked with $1 \%$ bovine serum albumin for $15 \mathrm{~min}$. After washing, the specimens were incubated with ani-AQP2 antibody for 45 min at $37{ }^{\circ} \mathrm{C}$. Then, the specimens were exposed to secondary antibody, Alexa Fluor 488-conjugated chicken anti-rabbit IgG (cat. no. A31571; Invitrogen, Life Technologies, Carlsbad, CA, USA). Microscopic observation was performed using a confocal microscope (Fluoview FV300, Olympus, Tokyo, Japan).

\subsection{Statistical Analysis}

The data are shown as a box plot. Individual values are represented as dots. Also, the mean \pm SEM values are shown in supplementary tables (Tables S2 and S4-S6).

Statistical comparisons were accomplished by Mann-Whitney U test. $p$ values $<0.05$ were considered statistically significant. 


\section{Results}

\subsection{Kidney Injury After Treatment with Cisplatin}

The summarized data for body weight, urine volume, plasma creatinine, and urea nitrogen concentrations are shown in Figure 1 and Table S2. Although body weight in the control group was not altered during the experimental period, that in the cisplatin group gradually decreased. Urine volume in the cisplatin group significantly decreased at $72 \mathrm{~h}$ and increased at $120 \mathrm{~h}$ in comparison with the control group. Cisplatin caused significant increases in plasma creatinine and urea nitrogen concentrations at $72 \mathrm{~h}, 120 \mathrm{~h}$, and $168 \mathrm{~h}$, and the highest values were observed at $120 \mathrm{~h}$. On the other hand, at $24 \mathrm{~h}$, cisplatin had no significant effect on the plasma creatinine concentration, although it caused a small but significant increase in the plasma urea nitrogen concentration. However, both values were within the normal range for rats, based on data provided by the laboratory animal production and supply company (Japan SLC Inc., http:/ /jslc.co.jp/pdf/rat/001_SD2013.pdf) (Figure 1C,D).
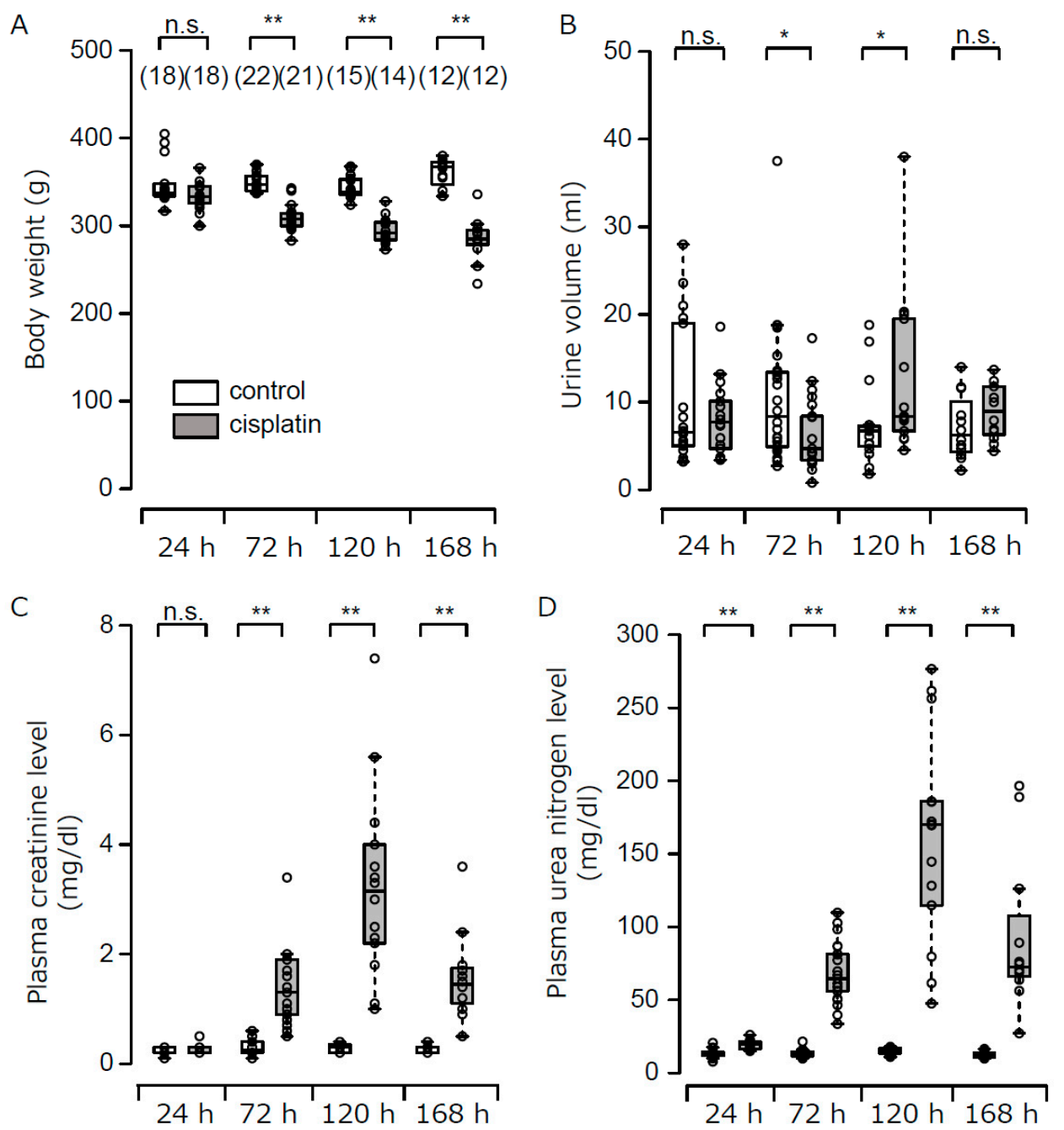

Figure 1. Time course of changes in body weight, urine volume, plasma creatinine and urea nitrogen concentrations after cisplatin treatment. Body weight (A), urine volume (B), plasma creatinine (C), and urea nitrogen concentrations (D) at $24 \mathrm{~h}, 72 \mathrm{~h}, 120 \mathrm{~h}$, and $168 \mathrm{~h}$ after treatment with either vehicle or cisplatin are shown. Numbers in parentheses indicate the number of animals subjected to experiments. ${ }^{*} p<0.05,{ }^{* *} p<0.01$, vs control rats. n.s. represents no significance.

As shown in Figure 2B, no histological change was observed in the outer medulla of the cisplatin group at $24 \mathrm{~h}$ after the treatment. However, vacuolization of proximal tubular cells and 
disruption of brush border were observed at $72 \mathrm{~h}$ (Figure 2C). At $120 \mathrm{~h}$, cisplatin caused loss of brush border, tubular cell necrosis, occlusion of tubular lumen by necrotic cell debris and casts, and cell infiltration in the interstitial region (Figure 2D). At $168 \mathrm{~h}$, in addition to a loss of brush border, cellular infiltration, and necrotic cell debris, remarkable dilation of tubular lumen and tubular cell regeneration (arrowheads in Figure 2F) were also observed in the outer medulla (Figure 2E,F). In the inner medulla, treatment with cisplatin for $24 \mathrm{~h}$ had little effect on the histology (Figure $2 \mathrm{H}$ ). However, at $168 \mathrm{~h}$ after the treatment, tubule dilation and cast formation were evident, especially in the loop of Henle (Figure 2I). Also, cells showing regeneration and mitosis (arrowheads in Figure 2I) were observed in the inner medulla.

\section{A OM control $24 \mathrm{~h}$}

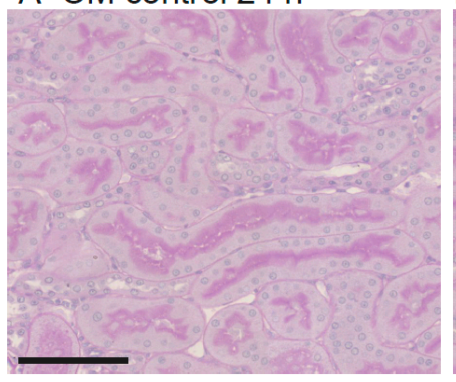

D OM cisplatin $120 \mathrm{~h}$

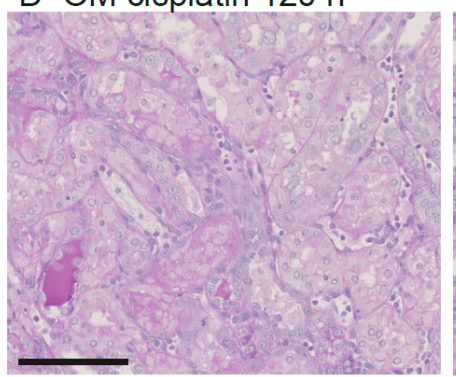

G IM control $24 \mathrm{~h}$

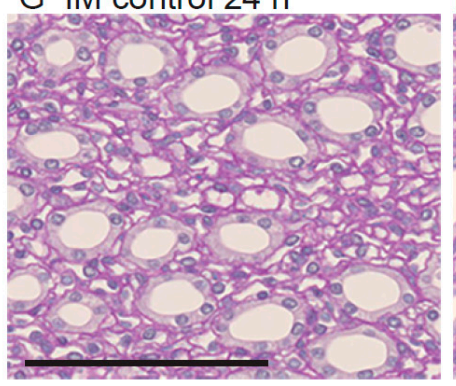

\section{B OM cisplatin $24 \mathrm{~h}$}

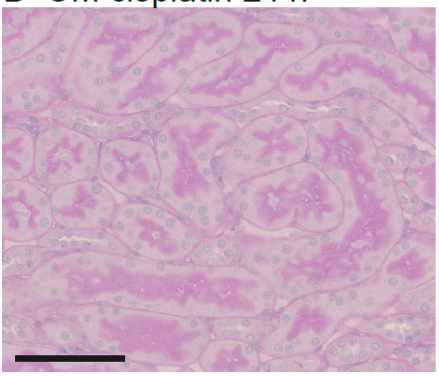

E OM cisplatin $168 \mathrm{~h}$

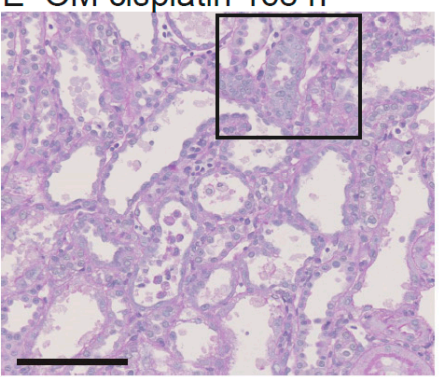

H IM cisplatin $24 \mathrm{~h}$

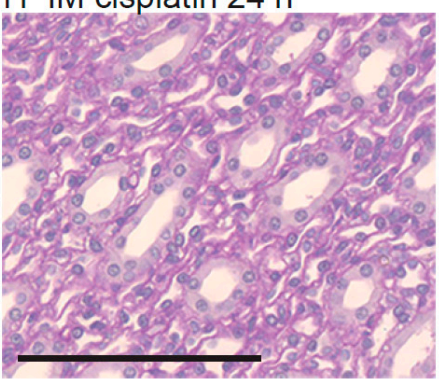

\section{OM cisplatin $72 \mathrm{~h}$}

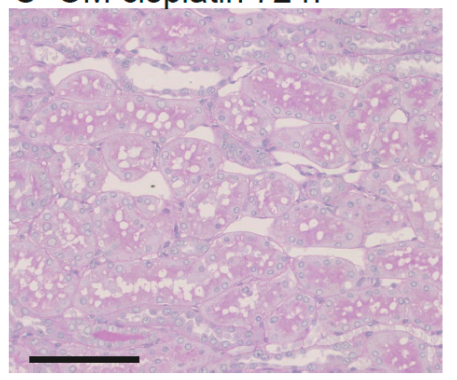

F OM cisplatin $168 \mathrm{~h}$

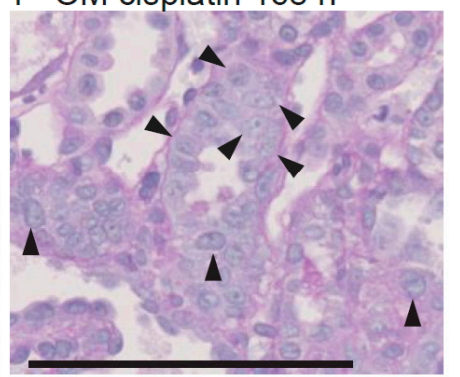

I IM cisplatin $168 \mathrm{~h}$

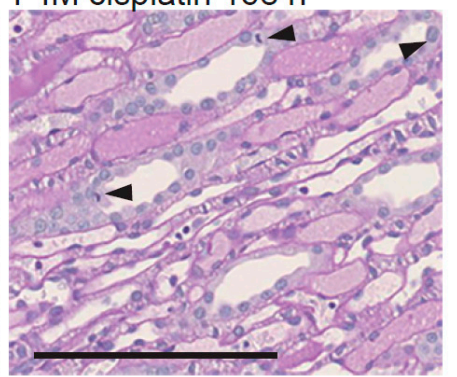

Figure 2. Renal histology after treatment with cisplatin. The outer (A-F) and inner medulla (G-I) regions of kidney sections stained with periodic acid-Schiff (PAS) from a rat in the control group at $24 \mathrm{~h} \mathrm{(A)} \mathrm{and} \mathrm{(G)} \mathrm{and} \mathrm{from} \mathrm{rats} \mathrm{in} \mathrm{the} \mathrm{cisplatin} \mathrm{group} \mathrm{at} 24 \mathrm{~h} \mathrm{(B)}$ and (H), $72 \mathrm{~h} \mathrm{(C),} 120 \mathrm{~h}(\mathbf{D})$, and $168 \mathrm{~h}$ (E), (F) and (I) are shown. These pictures are representative of three individuals at each time point and treatment. The high-magnification image of the box in $(\mathbf{E})$ is shown in (F). Arrow heads indicate the regenerating or mitotic tubular cells. Bars $=100 \mu \mathrm{m}$.

\subsection{Release of $u E V-A Q P 1$ and $-A Q P 2$ after Treatment with Cisplatin}

Next, we examined the release of $\mathrm{uEV}-\mathrm{AQP} 1$ after cisplatin treatment. We first measured urinary creatinine excretion in the control and cisplatin groups, and the mean \pm SEM values were shown in Table S3. In comparison with the control group, cisplatin had no significant effect on the excretion of creatinine at $24 \mathrm{~h}, 120 \mathrm{~h}$, and $168 \mathrm{~h}$. At $72 \mathrm{~h}$, cisplatin caused a slight decrease in the excretion by approximately $70 \%$. These data suggested that when the level of urinary creatinine was used to 
normalize the level of protein in the $\mathrm{uEV}$, this normalization method was appropriate at the $24 \mathrm{~h}, 120 \mathrm{~h}$, and $168 \mathrm{~h}$ time points. Also at $72 \mathrm{~h}$, even if a normalization error was present, in most cases, it would have been less than $30 \%$. Furthermore, this contamination error would have led to high estimates of the protein levels in the $\mathrm{uEV}$. When the data show a significant decrease in the level of protein in the $\mathrm{uEV}$, it is considered that the real value would be lower.

As shown in Figure 3A,B (Table S4), release of uEV-AQP1 in the cisplatin group was significantly increased at $24 \mathrm{~h}$. At $72 \mathrm{~h}$ and $120 \mathrm{~h}$, the difference between the control and cisplatin groups was not significant. At $168 \mathrm{~h}$, release of uEV-AQP1 was significantly decreased.

Figure 3C,D (Table S4) summarizes the data for uEV-AQP2. Release of uEV-AQP2 in the cisplatin group was significantly decreased even at $24 \mathrm{~h}$, and the decrease was maintained during the experimental period.
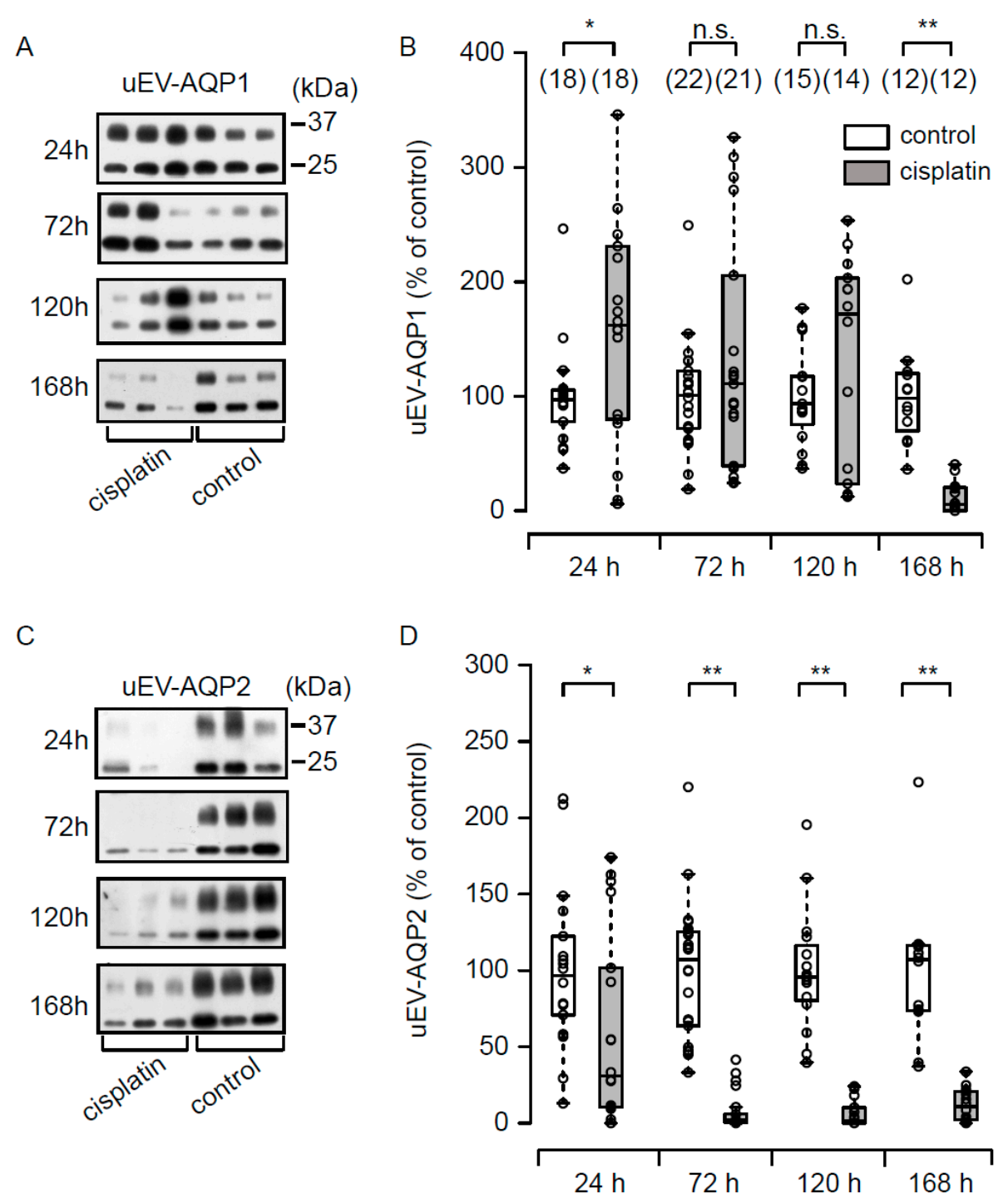

Figure 3. Release of aquaporin-1 in urinary extracellular vesicles (uEV-AQP1) and -AQP2 after cisplatin treatment. Typical immunoblots for uEV-AQP1 (A) and uEV-AQP2 (C) are shown. The summarized quantified data of immunoblot analyses of uEV-AQP1 (B) and uEV-AQP2 (D) are shown as boxplot graphs. Each value is indicated as a percentage of the mean value of uEV-AQP1 or -AQP2 level in the control rats at each time point. ${ }^{*} p<0.05,{ }^{* *} p<0.01$, vs control rats. n.s. represents no significance. The loading volume for immunoblot was normalized to urinary creatinine content. 


\subsection{Renal Expression of $A Q P 1$ and $A Q P 2$ after Treatment with Cisplatin}

Renal expression level of AQP1 was evaluated by immunoblotting, and the results are shown in Figure 4 (Table S5). Expression levels of AQP1 in the cortex and inner medulla in the cisplatin group were not significantly different from those in the control group at all of the time points examined in this study. On the other hand, in the outer medulla, cisplatin significantly increased expression of AQP1 at $24 \mathrm{~h}$ and decreased it at $168 \mathrm{~h}$.
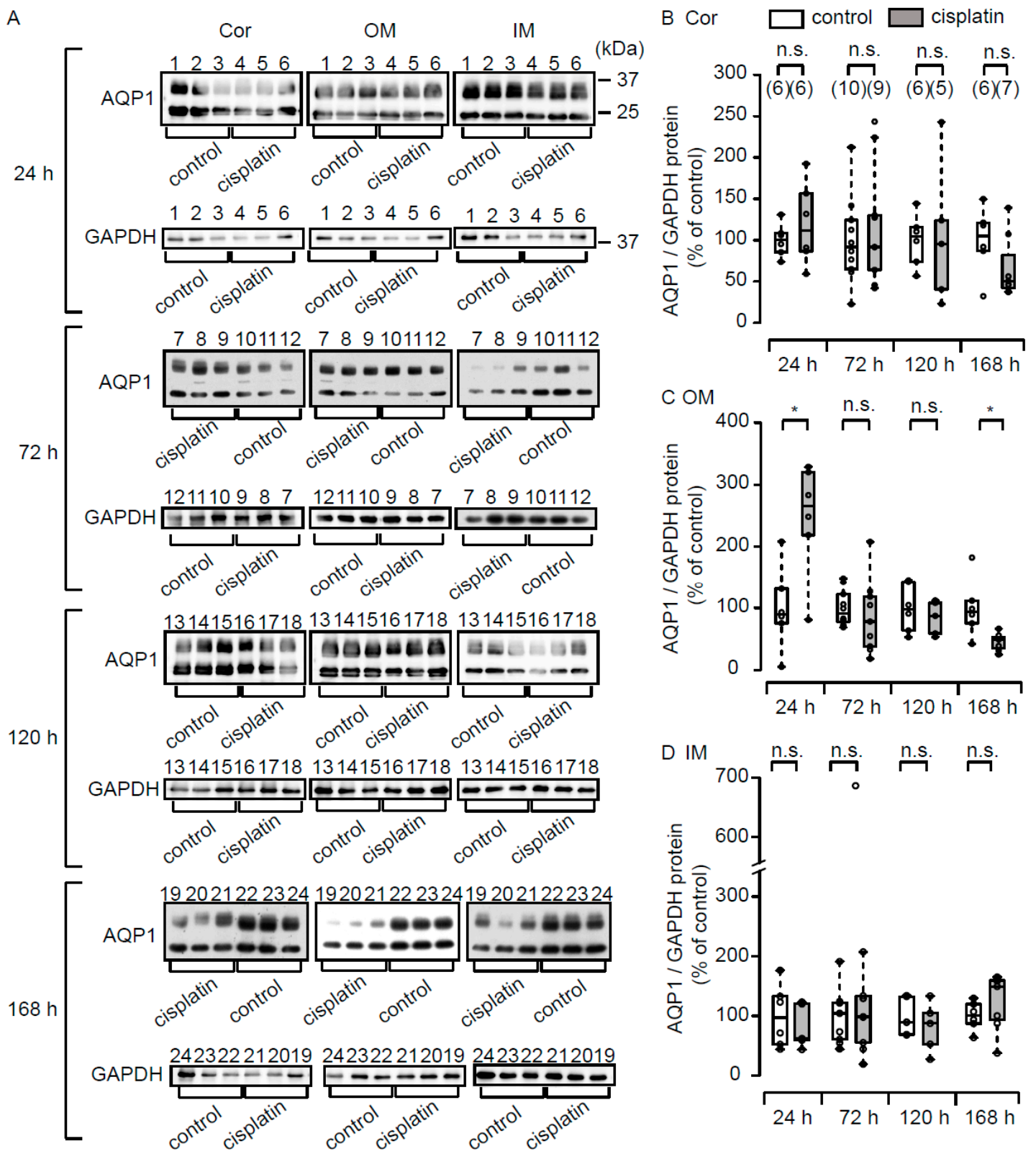

Figure 4. Renal AQP1 expression after cisplatin treatment. Representative immunoblots of renal AQP1 and GAPDH (A) and the quantified data for the cortex (Cor) (B), the outer medulla (OM) (C), and the inner medulla (IM) (D) are shown. Aquaporin-1 protein levels were normalized with corresponding GAPDH levels. Each value is expressed as percentage of mean value of the AQP1 levels in the control group at the corresponding time points. ${ }^{*} p<0.05$, vs control rats. n.s. represents no significance. The number above each lane indicates the individual rat number. Each lane was loaded with the same amount of total protein in the same gel. 
Figure 5 (Table S6) summarizes the data for renal expression of AQP2. In the cortex, expression of AQP2 was significantly decreased at $72 \mathrm{~h}$ and $120 \mathrm{~h}$ in the cisplatin group in comparison with the control group. In contrast to these decreases, at $168 \mathrm{~h}$ cisplatin up-regulated the expression of AQP2. In the outer medulla, cisplatin had little effect on the expression of AQP2 during the experimental period. In the inner medulla, expression of AQP2 was significantly increased at $24 \mathrm{~h}$. On the other hand, the expression was decreased at $72 \mathrm{~h}, 120 \mathrm{~h}$ and $168 \mathrm{~h}$, and the difference at $120 \mathrm{~h}$ was significant.

A
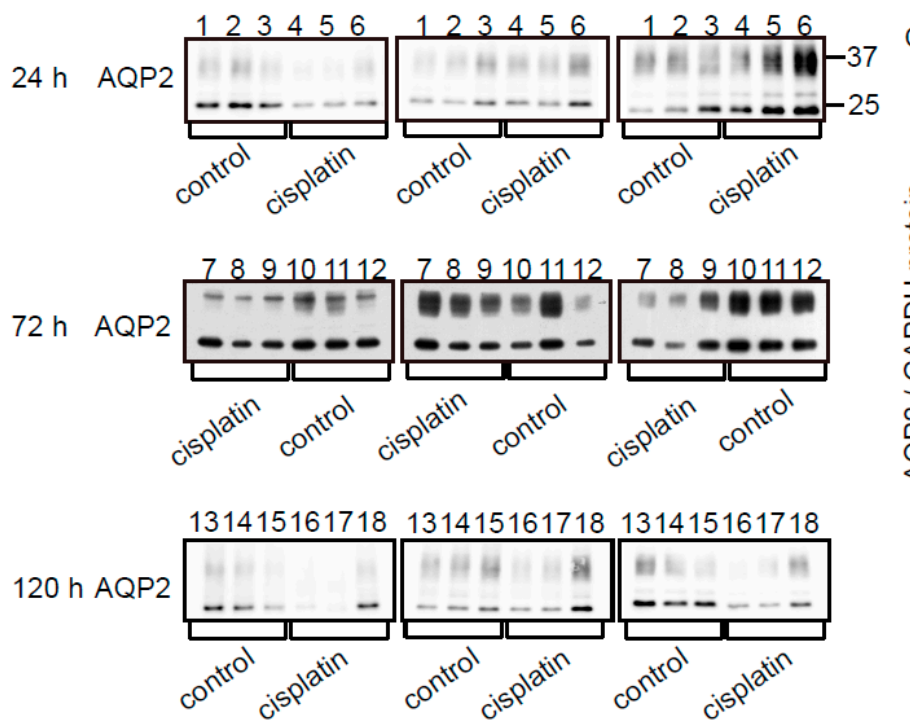

\section{4}

168 h AQP2

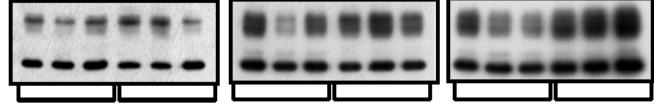

B $\square$ control $\square$ cisplatin

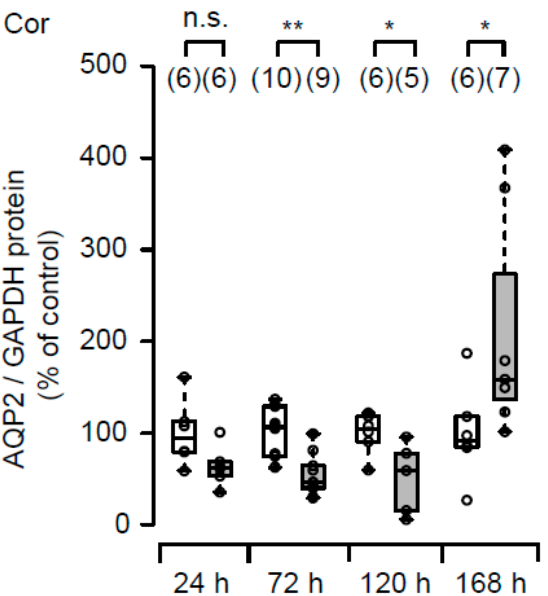

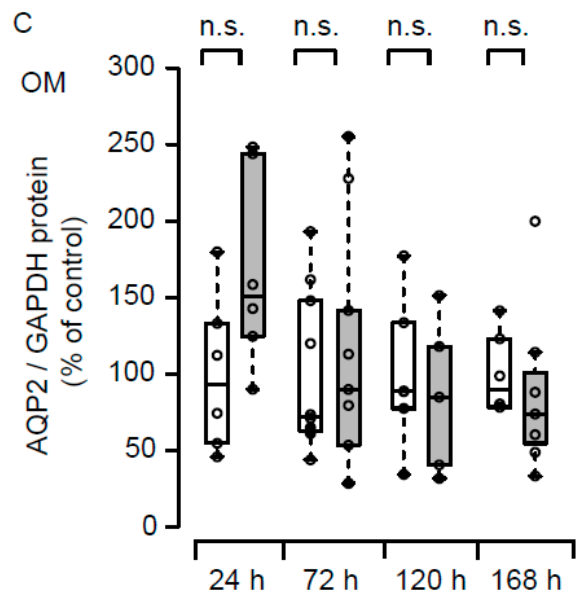

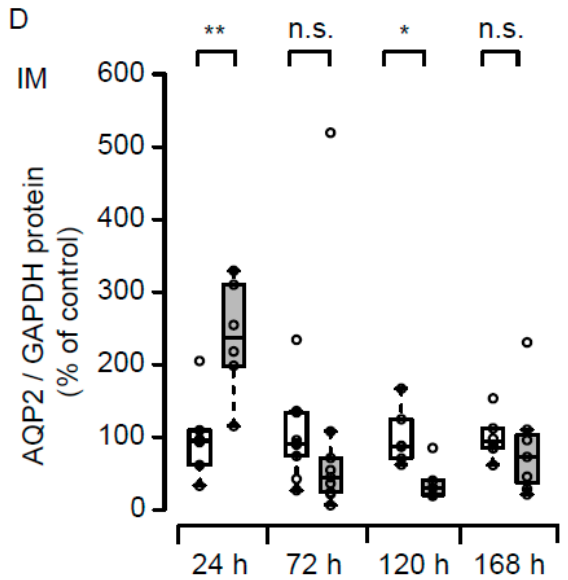

Figure 5. Renal AQP2 expression after cisplatin treatment. Representative immunoblots of renal AQP2 (A) and the quantified data for the cortex (Cor) (B), the outer medulla (OM) (C), and the inner medulla (IM) (D) are shown. Aquaporin-2 protein levels were normalized with corresponding GAPDH levels (shown in Figure 4). Each value is expressed as percentage of mean value of the AQP2 levels in the control group at the corresponding time points. ${ }^{*} p<0.05,{ }^{* *} p<0.01$, vs control rats. The number above each lane indicates the individual rat number. n.s. represents no significance. Each lane was loaded with the same amount of total protein in the same gel. 
As mentioned earlier, release of uEV-AQP2 was markedly decreased at $24 \mathrm{~h}$ after cisplatin treatment, even though the histological change was minimal. Therefore, we performed an immunofluorescence study using a sample from a rat that exhibited lower release of uEV-AQP2 (10.3\% relative to the control). As shown in Figure 6, there were no marked differences in the expression of AQP2 in the cortex and outer medulla between the control and cisplatin groups. In the inner medulla, expression of AQP2 was somewhat increased at $24 \mathrm{~h}$ after treatment with cisplatin, especially in the intracellular region.
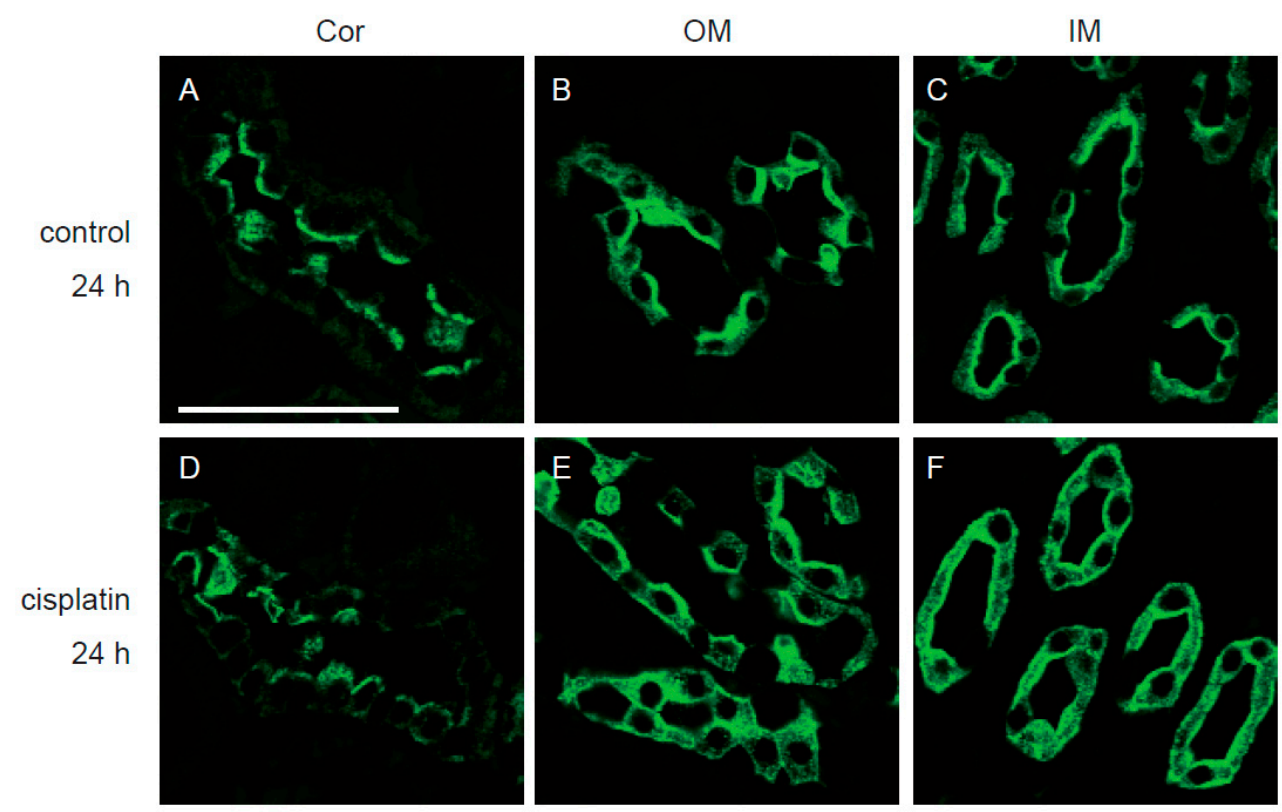

Figure 6. Immunofluorescence examination of renal AQP2 at $24 \mathrm{~h}$ after cisplatin treatment. The cortex (A) and (D), outer medulla (B) and (E), and inner medulla (C) and (F) regions of kidney sections stained with anti-AQP2 antibody at $24 \mathrm{~h}$ after treatment with either vehicle $(\mathbf{A}-\mathbf{C})$ or cisplatin $(\mathbf{D}-\mathbf{F})$. Bar $=50 \mu \mathrm{m}$.

\section{Discussion}

In the present study, the plasma creatinine and urea nitrogen concentrations were within the normal range at $24 \mathrm{~h}$ after cisplatin treatment. Thereafter, these concentrations increased progressively until $120 \mathrm{~h}$, and then were decreased somewhat at $168 \mathrm{~h}$. In accordance with these alterations, histological analyses showed that renal injury was minimal at $24 \mathrm{~h}$, whereas at $120 \mathrm{~h}$ loss of brush border, tubule cell necrosis, occlusion of the tubule lumen, and cell infiltration in the interstitial region were observed. At $168 \mathrm{~h}$ these pathological changes were still evident, and also cells characterized by a large nucleus, having an irregular size and shape, and mitotic figures were observed, suggesting that some of them were in the growth phase. This time course of cisplatin-induced renal injury corroborated previous reports [19]. During this experimental period, release of uEV-AQP1 was significantly increased at $24 \mathrm{~h}$, and thereafter returned to the control level. At $168 \mathrm{~h}$, release of uEV-AQP1 was markedly decreased. On the other hand, release of uEV-AQP2 was significantly decreased even at $24 \mathrm{~h}$ and this lower level was maintained until $168 \mathrm{~h}$. These results suggest that release of uEV-AQP2 might be a better indicator of early to late renal injury after cisplatin treatment. Furthermore, $\mathrm{uEV}-\mathrm{AQP} 1$ and $\mathrm{uEV}-\mathrm{AQP} 2$ in combination may reflect the later phase of cisplatin-induced kidney injury more accurately.

An interesting observation in this study was the early and continuous decrease in the release of uEV-AQP2 after cisplatin treatment. It has been suggested that the level of uEV-AQP2 is regulated by the abundance of its renal protein. For example, in rats with gentamicin-induced nephrotoxicity [8], release of uEV-AQP2 was significantly reduced, accompanied by a significant reduction of its renal expression. In the present study, expression of AQP2 was decreased in the cortex at $72 \mathrm{~h}$ and $120 \mathrm{~h}$, 
and in the inner medulla at $120 \mathrm{~h}$, after cisplatin treatment. Therefore, it is considered that the decreased renal expression in these regions is involved in the reduction of uEV-AQP2 release at $72 \mathrm{~h}$ and $120 \mathrm{~h}$ after cisplatin treatment. However, since cisplatin did not significantly reduce the expression of AQP2 protein at $24 \mathrm{~h}$, the decreased level of renal expression would not have explained the altered release of uEV-AQP2 at $24 \mathrm{~h}$. Besides the renal abundance of AQP2, it has been suggested that AVP may be another factor regulating the release of uEV-AQP2. Wen et al. [3] have shown that treatment of rats with desmopressin, an AVP analogue, caused a three- to four-fold increase in excretion of uEV-AQP2 in an apical-expression-dependent manner. Dear's group has reported that treatment of mCCDc11 cells, a kidney collecting duct cell line, with desmopressin increases the secretion of EV-AQP2 into the culture medium [20]. In rats, Clifton et al. have observed a decrease in the secretion of AVP from the posterior pituitary within $24 \mathrm{~h}$ after the cisplatin treatment [21]. Therefore, the inhibitory effect of cisplatin on the release of $\mathrm{uEV}-\mathrm{AQP} 2$ at $24 \mathrm{~h}$ may be due to reduced apical membrane expression of AQP2 via a decrease in the secretion of AVP. The increased intracellular expression of AQP2 we observed in the inner medulla (Figure 6F) may support this notion. Although it has been reported that direct measurement of the plasma AVP concentration is difficult, due to the association of AVP with platelets and the short half-life of plasma AVP, the measurement of a surrogate AVP marker, such as copeptin, might help to clarify the mechanism [22-24].

In the present study, we observed that release of uEV-AQP1 was significantly increased at $24 \mathrm{~h}$ and decreased at $168 \mathrm{~h}$ after cisplatin treatment. Along with these observations, cisplatin significantly increased the expression of AQP1 in the outer medulla at $24 \mathrm{~h}$ and decreased it at $168 \mathrm{~h}$. Because, similarly to AQP2, renal expression of AQP1 has also been reported to contribute to the release of $\mathrm{uEV}-\mathrm{AQP1}$ [9], alteration in the release of $\mathrm{uEV}-\mathrm{AQP1}$ is thought to be mediated through changes in its level of expression in the outer medulla.

We showed that expression of AQP1 in the outer medulla was significantly decreased and that of AQP2 in the inner medulla tended to be decreased at $168 \mathrm{~h}$ after cisplatin treatment. Similar observations have been made in the later phase of many types of renal injury models, including ischemia/reperfusion and gentamicin-treatment models [8-10]. In this phase, cell proliferation is thought to repair the injury sites. In fact, in the present study, we observed some cells in the growth phase at this time point. Also, markers of cell proliferation, including proliferating cell nuclear antigen, are reportedly expressed in a similar phase in other experimental models of renal injury [25,26]. On the other hand, in growing renal cells, it has been considered that expression of renal functional proteins such as transporters and AQPs are down-regulated [27,28]. Therefore, the increase in the number of proliferative cells may result in reduction of the renal expression of AQP1 and AQP2 at a later phase of cisplatin-induced kidney injury.

\section{Conclusions}

We have found that cisplatin affects the release of $\mathrm{uEV}-\mathrm{AQP} 1$ and -AQP2, and that examination of these $\mathrm{uEV}$-AQPs in combination may allow early to late detection of cisplatin-induced renal impairment. However, as studies on the regulation of uEV-AQP1 and -AQP2 release are still in their early stages, further work to identify the molecular mechanism underlying their release will be necessary.

Supplementary Materials: The supplementary materials are available online http:/ /www.mdpi.com/2073-4409/ 8/2/139/s1.

Author Contributions: H.S. and M.I. conceived and designed research; H.S., S.O.-H. and M.I. performed experiments; H.S., S.O.-H. and M.I. analyzed data; H.S., S.O.-H. and M.I. interpreted results of experiments; H.S., S.O.-H. and M.I. prepared figures; H.S., S.O.-H. and M.I. drafted manuscript; H.S., S.O.-H. and M.I. approved final version of manuscript.

Funding: The work is supported by JSPS KAKENHI, 25660241 (M.I.), 25221205 (M.I.), 15H04594 (M.I.), 16 K15047 (M.I.), 18H02348 (M.I.), 15K18784 (H.S.), and 18K05996 (H.S.).

Conflicts of Interest: No conflicts of interest, financial or otherwise, are declared by the authors. 


\section{References}

1. Nielsen, S.; Frøkiaer, J.; Marples, D.; Kwon, T.H.; Agre, P.; Knepper, M.A. Aquaporins in the kidney: From molecules to medicine. Physiol. Rev. 2002, 82, 205-244. [CrossRef] [PubMed]

2. Ikeda, M.; Matsuzaki, T. Regulation of aquaporins by vasopressin in the kidney. Vitam. Horm. 2015, 98, 307-337. [PubMed]

3. Wen, H.; Frokiaer, J.; Kwon, T.H.; Nielsen, S. Urinary excretion of aquaporin-2 in rat is mediated by a vasopressin-dependent apical pathway. J. Am. Soc. Nephrol. 1999, 10, 1416-1429.

4. Pisitkun, T.; Shen, R.F.; Knepper, M.A. Identification and proteomic profiling of exosomes in human urine. Proc. Natl. Acad. Sci. USA 2004, 101, 13368-13373. [CrossRef] [PubMed]

5. Merchant, M.L.; Rood, I.M.; Deegens, J.K.J.; Klein, J.B. Isolation and characterization of urinary extracellular vesicles: Implications for biomarker discovery. Nat. Rev. Nephrol. 2017, 13, 731-749. [CrossRef] [PubMed]

6. Corbetta, S.; Raimondo, F.; Tedeschi, S.; Syrèn, M.L.; Rebora, P.; Savoia, A.; Baldi, L.; Bettinelli, A.; Pitto, M. Urinary exosomes in the diagnosis of Gitelman and Bartter syndromes. Nephrol. Dial. Transplant. 2015, 30, 621-630. [CrossRef] [PubMed]

7. Miyazawa, Y.; Mikami, S.; Yamamoto, K.; Sakai, M.; Saito, T.; Yamamoto, T.; Ishibashi, K.; Sasaki, S. AQP2 in human urine is predominantly localized to exosomes with preserved water channel activities. Clin. Exp. Nephrol. 2018, 22, 782-788. [CrossRef]

8. Abdeen, A.; Sonoda, H.; El-Shawarby, R.; Takahashi, S.; Ikeda, M. Urinary excretion pattern of exosomal aquaporin-2 in rats that received gentamicin. Am. J. Physiol. Renal Physiol. 2014, 307, F1227-F1237. [CrossRef]

9. Sonoda, H.; Yokota-Ikeda, N.; Oshikawa, S.; Kanno, Y.; Yoshinaga, K.; Uchida, K.; Ueda, Y.; Kimiya, K.; Uezono, S.; Ueda, A.; et al. Decreased abundance of urinary exosomal aquaporin-1 in renal ischemia-reperfusion injury. Am. J. Physiol. Renal Physiol. 2009, 297, F1006-F1016. [CrossRef]

10. Asvapromtada, S.; Sonoda, H.; Kinouchi, M.; Oshikawa, S.; Takahashi, S.; Hoshino, Y.; Sinparadeelerdkul, T.; Yokota-Ikeda, N.; Matsuzaki, T.; Ikeda, M. Characterization of urinary exosomal release of aquaporin-1 and -2 after renal ischemia-reperfusion in rats. Am. J. Physiol. Renal Physiol. 2018, 314, F584-F601. [CrossRef]

11. Rabik, C.A.; Dolan, M.E. Molecular mechanisms of resistance and toxicity associated with platinating agents. Cancer Treat. Rev. 2007, 33, 9-23. [CrossRef] [PubMed]

12. Pabla, N.; Dong, Z. Cisplatin nephrotoxicity: Mechanisms and renoprotective strategies. Kidney Int. 2008, 73, 994-1007. [CrossRef] [PubMed]

13. Gonzales-Vitale, J.C.; Hayes, D.M.; Cvitkovic, E.; Sternberg, S.S. The renal pathology in clinical trials of cis-platinum (II) diamminedichloride. Cancer 1977, 39, 1362-1371. [CrossRef]

14. Dentino, M.; Luft, F.C.; Yum, M.N.; Williams, S.D.; Einhorn, L.H. Long term effect of cis-diamminedichloride platinum (CDDP) on renal function and structure in man. Cancer 1978, 41, 1274-1281. [CrossRef]

15. Brady, H.R.; Zeidel, M.L.; Kone, B.C.; Giebisch, G.; Gullans, S.R. Differential actions of cisplatin on renal proximal tubule and inner medullary collecting duct cells. J. Pharmacol. Exp. Ther. 1993, 265, 1421-1428. [PubMed]

16. Schwerdt, G.; Freudinger, R.; Schuster, C.; Weber, F.; Thews, O.; Gekle, M. Cisplatin-induced apoptosis is enhanced by hypoxia and by inhibition of mitochondria in renal collecting duct cells. Toxicol. Sci. 2005, 85, 735-742. [CrossRef]

17. Kishore, B.K.; Krane, C.M.; Di Iulio, D.; Menon, A.G.; Cacini, W. Expression of renal aquaporins 1, 2, and 3 in a rat model of cisplatin-induced polyuria. Kidney Int. 2000, 58, 701-711. [CrossRef]

18. Kim, S.W.; Lee, J.U.; Nah, M.Y.; Kang, D.G.; Ahn, K.Y.; Lee, H.S.; Choi, K.C. Cisplatin decreases the abundance of aquaporin water channels in rat kidney. J. Am. Soc. Nephrol. 2001, 12, 875-882.

19. Dobyan, D.C.; Levi, J.; Jacobs, C.; Kosek, J.; Weiner, M.W. Machanism of cis-platinum nephrotoxicity: II. Morphologic observations. J. Pharmacol. Exp. Ther. 1980, 213, 551-556.

20. Street, J.M.; Brikhoff, W.; Menzies, R.I.; Webb, D.J.; Baily, M.A.; Dear, J.W. Exoaomal transmission of functional aquaporin 2 in kidney cortical collecting duct cells. J. Physiol. 2011, 589, 6119-6127. [CrossRef]

21. Clifton, G.G.; Pearce, C.; O’Neill, W.M., Jr.; Wallin, J.D. Early polyuria in the rat following single-dose cis-dichlorodiammineplatinum (II): Effects on plasma vasopressin concentration and posterior pituitary function. J. Lab. Clin. Med. 1982, 100, 659-670. [PubMed]

22. Preibisz, J.J.; Sealey, J.E.; Laragh, J.H.; Cody, R.J.; Weksler, B.B. Plasma and platelet vasopressin in essential hypertension and congestive heart failure. Hypertension 1983, 5, I129-I138. [CrossRef] [PubMed] 
23. Baumann, G.; Dingman, J.F. Distribution, blood transport, and degradation of antidiuretic hormone in man. J. Clin. Invest. 1976, 57, 1109-1116. [CrossRef]

24. Morgenthaler, N.G.; Struck, J.; Alonso, C.; Bergmann, A. Assay for the measurement of copeptin, a stable peptide derived from the precursor of vasopressin. Clin. Chem. 2006, 52, 112-119. [CrossRef]

25. Fujigaki, Y.; Sakakima, M.; Sun, Y.; Fujikura, T.; Tsuji, T.; Yasuda, H.; Hishida, A. Cell division and phenotypic regression of proximal tubular cells in response to uranyl acetate insult in rats. Nephrol. Dial. Transplant. 2009, 24, 2686-2692. [CrossRef] [PubMed]

26. Trepiccione, F.; Capasso, G.; Nielsen, S.; Christensen, B.M. Evaluation of cellular plasticity in the collecting duct during recovery from lithium-induced nephrogenic diabetes insipidus. Am. J. Physiol. Renal Physiol. 2013, 305, F919-F929. [CrossRef]

27. Shioda, T.; Ohta, T.; Isselbacher, K.J.; Rhoads, D.B. Differentiation-dependent expression of the Na+/glucose cotransporter (SGLT1) in LLC-PK1 cells: Role of protein kinase C activation and ongoing transcription. Proc. Natl. Acad. Sci. USA 1994, 91, 11919-11923. [CrossRef]

28. Bonilla-Felix, M.; Jiang, W. Aquaporin-2 in the immature rat: Expression, regulation, and trafficking. J. Am. Soc. Nephrol. 1997, 8, 1502-1509.

(C) 2019 by the authors. Licensee MDPI, Basel, Switzerland. This article is an open access article distributed under the terms and conditions of the Creative Commons Attribution (CC BY) license (http:/ / creativecommons.org/licenses/by/4.0/). 